\title{
Simulation Studies of a Possible Multi-stage XFEL at ELETTRA
}

\author{
W.M. Fawley ${ }^{a}$, W.A. Barletta ${ }^{a}$, C.J. Bocchetta ${ }^{b}$, and R. Bonifacio ${ }^{c}$

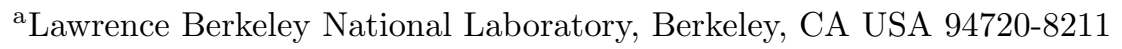 \\ ${ }^{\mathrm{b}}$ Sincrotrone Trieste, S.C.p.A, S.S. 14km, 34012 Trieste, Italy \\ ${ }^{\mathrm{c} U n i v e r s i t a}$ degli Studi di Milano, 20133 Milano, Italy
}

Presently there is strong interest in developing a 4th generation light source at VUV and soft x-ray wavelengths at the ELETTRA facility at Trieste. One proposal centers around using the existing linac at $1.0 \mathrm{GeV}$ energy with a new photocathode and bunch compression to achieve an output beam at 600 Amp current, 2-4 mm-mrad normalized emittance, and $0.05 \%$ instantaneous energy spread. To achieve output radiation in the 10- to 40-nm wavelength region, we consider a multi-stage device which is initiated by a coherent seed laser operating at 200 $\mathrm{nm}$. We present numerical simulations of various undulator/optical-klystron configurations, seeking to optimize the overall output power level while minimizing the total length of undulator sections needed. Our results suggest multi-MW instantaneous powers are possible at $10-\mathrm{nm}$ wavelengths.

\section{Introduction}

Over the past decade there has been continually growing interest in basing so-called 4th generation light sources upon x-ray FEL's using the principle of self-amplification of spontaneous emission (SASE). Since this approach to short wavelength generation depends upon the amplification of electron beam shot noise, there is an inherent statistical fluctuation both in the power level and spectral content of a SASE FEL's output radiation. Equivalently, the longitudinal coherence is quite limited.

Working along side of the SASE bandwagon rush, others (see, e.g. $\mathrm{Yu}[1]$ ) have suggested an alternative approach of using a harmonic cascade FEL to reach the VUV-XUV spectral region. This approach utilizes the long-known property (see, e.g. Bonifacio et al.[2]) of high gain FEL amplifiers that microbunching at higher harmonics grow nonlinearly due to coupling with the fundamental. While the cascade approach is certainly more complex than a single SASE undulator, it has a number of important advantages: (1) longitudinal coherence of the output radiation is initially determined by properties of the master oscillator laser and should not degrade significantly so long as both the effective shot noise power level remains proportionally small in each stage and there are no significant high frequency temporal variations in electron beam properties (2) shotto-shot fluctuations depend mainly upon macroscopic beam properties, not uncontrollable microscopic noise distributions (3) depending upon experimental timing control of the e-beam and the external input laser, one (in principle at least) can obtain extremely short output pulses.

Following a planned upgrade to the storage ring at the ELETTRA facility at Trieste, the existing $1.0-\mathrm{GeV}$ energy linac will become available for new applications. One possibility is to add both a new photocathode injector and an output electron beam compressor to then produce a high quality beam for use in a multi-stage, harmonic cascade FEL which could reach output wavelengths in the $10-$ to $40-\mathrm{nm}$ spectral range. The cascade would consist of several modules, each containing a "modulator" undulator which strongly microbunches the electron beam, followed by a "radiator" whose undulator magnetic strength is tuned for radiation output at a higher harmonic of the fundamental resonant wavelength of the upstream modulator. In many cases one might also use a chromatic dispersive element between the modulator and radiator undulators (i.e. an optical klystron configuration) to 
Table 1

Harmonic Cascade Undulator/FEL Parameters

\begin{tabular}{llll}
\hline & \multicolumn{1}{c}{$\mathrm{U} 1$} & \multicolumn{1}{c}{$\mathrm{U} 2$} & $\mathrm{U} 3$ \\
\hline$\lambda_{s}(\mathrm{~nm})$ & 200 & 40 & 10 \\
$\lambda_{w}(\mathrm{~mm})$ & 64.4 & 44.5 & 44.5 \\
$a_{w}$ & 4.755 & $2.4175,2.4180$ & 0.838 \\
$B_{w}(\mathrm{~T})$ & 0.79 & 0.58 & 0.20 \\
FEL parameter $\rho$ & $3.5 \times 10^{-3}$ & $2.5 \times 10^{-3}$ & $1.3 \times 10^{-3}$ \\
$L_{w}(\mathrm{~m})$ & 5.6 & $17.52,19.52$ & 11.06 \\
drift section length $(\mathrm{m})$ & 0.8 & 0.92 & 0.766 \\
$P_{\text {out }}(\mathrm{MW})$ & 106 & 510,1400 & 2.3 \\
output $\left|b_{1}\right|$ & 0.35 & $0.19,0.26$ & 0.011 \\
$\sigma_{\gamma, \text { out }}$ & 2.2 & $4.8,5.5$ & 4.8 \\
\hline
\end{tabular}

increase the microbunching without further increasing the instantaneous energy spread (which significantly degrades the gain at the shortest wavelengths).

In this paper, we present the results of numerical simulations with the GINGER simulation code[3] of sample ELETTRA harmonic cascade configurations, seeking to optimize the overall output power level while minimizing the total length. Another paper in these proceedings [4] gives additional details concerning the ELETTRA XUV FEL proposal.

Our FEL configuration consists of 3 undulators (unimaginatively named $\mathrm{U} 1, \mathrm{U} 2$, and $\mathrm{U} 3$ ), whose resonant wavelengths correspond to 200-, 40-, and 10-nm, respectively. Table 1 gives their physical parameters together with some FEL output characteristics. In each case the undulators are APPLE-II type, presumed to have helical polarization, and are composed of 2-m sections separated by periodic drift/focusing/diagnostic sections whose lengths were chosen to retain phase synchronization between the radiation and electron beam microbunching. We now give some additional details concerning the layout and performance of each harmonic stage.

\section{Basic Electron Beam and FEL Parame- ters}

For our numerical studies, we adopted the following electron beam parameters following a linac upgrade: $I_{B}=600 \mathrm{~A}, E_{B}=1.0 \mathrm{GeV}$, $\sigma_{\gamma}=1.0, \varepsilon_{N}=2.0 \pi \mathrm{mm}-\mathrm{mrad}$. The GINGER runs were done in time-steady, "monochromatic" mode (i.e. no slippage, shot noise, or sideband effects) in which the $6 \mathrm{D}$ macroparticle distribution from one undulator is passed directly to the next. Although the code does not at present selfconsistently include radiation emission at higher harmonics, (which should be small for a helicallypolarized undulator), it does diagnose the microbunching at higher harmonics. No fringe fields were included for the focusing quadrupoles and the dispersive section present at the end of the U1 undulator is treated as a simple energydependent kick in longitudinal phase $\theta$. Furthermore, each downstream undulator was presumed to butt firmly against its upstream neighbor i.e. there are no additional drift spaces other than the periodic ones present in each undulator. A more self-consistent study would need to include all of these phenomena and others (e.g. alignment errors) to be more fully believable.

In this paper, we presume that the full electron beam pulse undergoes FEL action in each undulator stage, unlike $\mathrm{Yu}[5]$ who in a similar paper which studies a harmonic cascade for ELETTRA 
presumes that the electron beam temporal jitter is sufficiently low that one may use a much temporally shorter master oscillator pulse. Low jitter allows one to use "fresh" portions (in which the instantaneous energy spread has not been increased by upstream FEL action) of the electron beam pulse in each downstream undulator, significantly increasing the gain and output power at shorter wavelengths.

\subsection{Stage 1: 200-nm Modulator}

The U1 undulator serves the purpose of strongly modulating the longitudinal density of the electron beam so that it will radiate strongly at the 5th harmonic (i.e. 40-nm) in the next undulator, U2. We adopted a 10-MW input power for the external input laser; the exact value is not crucial as smaller values can be compensated for by increasing the undulator length. For this FEL there is strong exponential gain but it is purposely stopped well before saturation in order to minimize the accompanying increase in energy spread. The radiation has an equilibrium rms (power) radius of $0.4 \mathrm{~mm}$ in contrast to the rms electron beam radius $\sigma_{r}$ of $0.11 \mathrm{~mm}$. At $z=4.8 \mathrm{~m}$ (the end of the second undulator section), we have placed a chromatic dispersive section (whose dispersion is equivalent to an additional $1.68 \mathrm{~m}$ of undulator) to further increase the microbunching (in this case $b_{1}$ increases from 0.21 to 0.35 and $b_{5}$ from 0.003 to 0.021 ) and a quadrupole doublet for better matching into U2. In GINGER, microbunching at harmonic $m$ is defined as

$$
b_{m} \equiv\left|\sum_{n=1}^{N P} \exp \left(i m \theta_{n}\right)\right| / N P
$$

with $N P$ being the number of macroparticles and $\theta$ being the longitudinal phase relative to a plane wave at the fundamental wavelength.

\subsection{Stage 2: 40-nm Radiator/Modulator}

The U2 undulator first serves as a "radiator" for the pre-bunched electron beam entering from the U1 modulator/dispersive section and then as a high gain amplifier which also strongly bunches the beam at 40-nm wavelength and higher harmonics. The equilibrium radiation mode size is $0.3 \mathrm{~mm}$ (corresponding to a Rayleigh range of

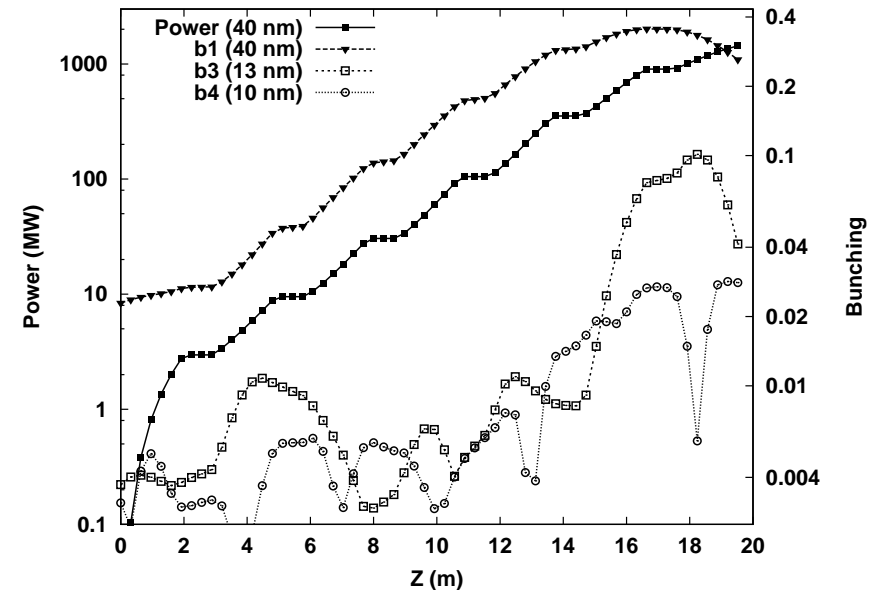

Figure 1. Radiation power at 40-nm and microbunching at 40-, 13-, and 10-nm wavelengths for the U2 undulator. On this semilog plot, the bunching scale is one-half that of the power to provide simple comparison of growth rates.

$3.5 \mathrm{~m}$ ). In addition to the normal undulator focusing, there are also quadrupole doublets in each drift section providing additional focusing to keep the electron beam $\sigma_{r} \sim 0.08 \mathrm{~mm}$. If the goal is maximum output power at $40-\mathrm{nm}$, a total length of $19.5 \mathrm{~m}$ (seven undulator sections) gives $1.4 \mathrm{GW}$ (2.3\% energy extraction) and a final rms energy spread of $\sigma_{\gamma}=5.5$. Tapering the undulator could provide even greater output power if wanted. In Fig. 1 we plot the $40-\mathrm{nm}$ radiation power and microbunching at $40-\mathrm{nm}$ and its harmonics versus $z$. The plotted results were optimized for peak 40-nm output. Stopping the undulator at $17.5 \mathrm{~m}$ (six sections) and optimizing $a_{w}$ for peak bunching rather than radiation power reduces the power to $0.5 \mathrm{GW}$ but provides much better downstream performance in U3 at 10-nm wavelength.

\subsection{Stage 3: 10-nm Radiator}

The U3 undulator serves as a radiator, converting the microbunching modulation produced by the U2 undulator into coherent $10-\mathrm{nm}$ radiation. Since the normalized instantaneous energy 


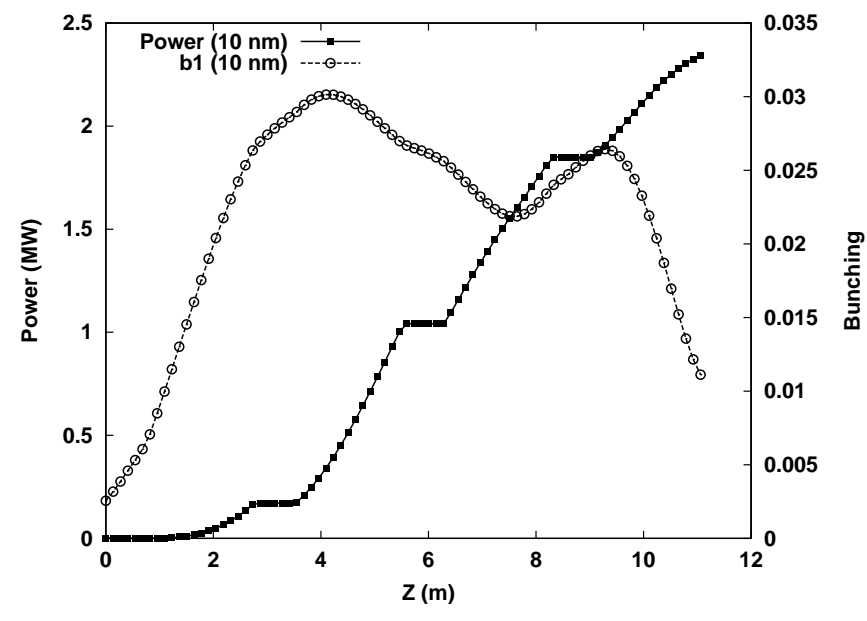

Figure 2. Radiation power and microbunching versus $z$ for the U3 undulator at $10-n m$ wavelength.

\section{Conclusions}

In this paper we have presented numerical simulations of a three-stage, harmonic cascade of a possible XUV FEL at ELETTRA. Beginning with 10-MW of external laser input at 200-nm, we showed that over $1 \mathrm{GW}$ of instantaneous radiation power is obtainable at $40-\mathrm{nm}$ and more than 2-MW at 10-nm wavelength. Our design was conservative in the sense that we presumed that temporal jitter of the linac beam would require modulating the full electron beam pulse at 200-nm and shorter wavelengths, with the consequence that the large energy spread present at the entrance of the third undulator prevents significant exponential gain at $\lambda_{s}=10 \mathrm{~nm}$. If this restriction were dropped, much greater powers would be possible and we expect that our results would parallel those of $\mathrm{Yu}[5]$. Our undulator parameters (see Table 1) are all sufficiently conservative that a linac upgrade to higher energies (e.g. $E_{B} \geq 1.25 \mathrm{GeV}$ ) would increase gain at the shorter wavelengths without encountering field strength problems in any of the undulator stages.

\section{Acknowledgements}

This work was supported at LBNL by the U.S. Department of Energy under Contract No. DEAC03-76SF0098. Computational resources were provided in part by the National Energy Research Supercomputer Center (NERSC).

\section{REFERENCES}

1. L.H. Yu, Phys. Rev A, 44, 5178 (1991).

2. R. Bonifacio et al., Nucl. Inst. Meth., A296, 787 (1990).

3. W.M. Fawley, LBNL Report No. LBNL49625, 2002.

4. C.J. Bocchetta et. al., Poster TH-P-04, "Overview of FERMI@ELETTRA a Proposed Ultra Bright Coherent X-ray Source in Italy", this conference.

5. L.H. Yu, Poster We-P-58, "Simulations for the FERMI@ELETTRA Proposal to Generate 40-nm and 10-nm Coherent Radiation Using the HGHG Scheme", this conference. 